\title{
Power-scaling performance of a three-dimensional tritium betavoltaic diode
}

\author{
Baojun Liu, ${ }^{1}$ Kevin P. Chen, ${ }^{1, a)}$ Nazir P. Kherani, ${ }^{2}$ and Stefan Zukotynski ${ }^{2}$ \\ ${ }^{1}$ Department of Electrical and Computer Engineering, University of Pittsburgh, \\ Pittsburgh, Pennsylvania 15261, USA \\ ${ }^{2}$ Department of Electrical and Computer Engineering, University of Toronto, \\ Toronto, Ontario M5S 3G4, Canada
}

(Received 13 June 2009; accepted 17 November 2009; published online 8 December 2009)

\begin{abstract}
Three-dimensional diodes fabricated by electrochemical etching are exposed to tritium gas at pressures from 0.05 to $33 \mathrm{~atm}$ at room temperature to examine its power scaling performance. It is shown that the three-dimensional microporous structure overcomes the self-absorption limited saturation of beta flux at high tritium pressures. These results are contrasted against the three-dimensional device powered in one instance by tritium absorbed in the near surface region of the three-dimensional microporous network, and in another by a planar scandium tritide foil. These findings suggest that direct tritium occlusion in the near surface of three-dimensional diode can improve the specific power production. (C) 2009 American Institute of Physics.
\end{abstract}

[doi:10.1063/1.3272105]

Utilization of radioisotope power sources has been explored since the 1950s. ${ }^{1}$ In light of recent progress in nanotechnology and micro/nanoelectromechanical systems (M/NEMS), radiovoltaic microsources have regained significant interest owing to their potential for providing uninterrupted energy for low-power consuming microsystems operating at microwatt level. ${ }^{1-4}$ Advances in nanotechnology also offer innovative opportunities to utilize radioisotope power sources more efficiently. Direct radiovoltaic devices utilizing semiconductor $p n$ junctions are well-suited to beta emitters such as tritium $\left({ }^{3} \mathrm{H}\right)$ and ${ }^{63} \mathrm{Ni}$, primarily due to minimum lattice damage considerations. These microbatteries are referred to as betavoltaics. A range of semiconductor materials have been explored for betavoltaics such as amorphous silicon, ${ }^{2}$ silicon carbide, ${ }^{3}$ and gallium arsenide, ${ }^{1}$ however, their power production and energy conversion efficiency based on conventional planar diodes have been limited, one reason of which is the limited available open surface area directly exposed to the energetic betaparticles. Recently, micro-engineering has been used to fabricate so-called threedimensional (3D) $p n$ diodes to considerably increase the surface area of a $p n$-junction device in a given volume. ${ }^{4,5}$ Porous or trenched 3D $p n$-junction fabricated using either electrochemical etching ${ }^{4}$ or deep reactive ion etching ${ }^{5}$ promise a significant increase in the surface to volume ratio of the device and accordingly increase in power production and energy conversion efficiency of nuclear batteries.

The 3D configuration is particularly advantageous for betavoltaic devices harnessing low energy beta particles. Tritium is suitable for betavoltaic applications owing to its inherent characteristics of relatively benign radiation, long half-life of 12.3 years, as well as its low cost. ${ }^{2,4,6}$ However, the low kinetic energy $\left(\mathrm{E}_{\mathrm{avg}}: 5.7 \mathrm{keV}\right)$ of tritium betas corresponds to a very short range in semiconductor materials; the majority of the kinetic energy is absorbed within $\sim 200 \mathrm{~nm}$. Hence, effective energy conversion of low-energy beta particles requires a large surface powering the betavoltaic device. The use of $3 \mathrm{D} p n$-junctions is one approach that can

${ }^{a)}$ Electronic mail: kchen@engr.pitt.edu. improve power production. Pores and ridges fabricated in semiconductor materials at the micron and submicron level permit large surface to volume ratios. Moreover, infiltration of micrometer sized porous structures with tritium fuel minimizes self-absorption losses in energy and results in relatively complete delivery of tritium beta energy into the energy conversion region of the device. Thus, high-density tritium fuel can be utilized efficiently.

In this letter, we examine the power scaling of 3D diodes as a function of tritium fuel density. By exposing 3D diodes to gaseous tritium, the power output of a 3D diode is studied from 0.05 to $33 \mathrm{~atm}$ tritium at room temperature, which corresponds to an isotope power density of $4.35 \mu \mathrm{W} / \mathrm{cm}^{3}$ to $2.87 \mathrm{~mW} / \mathrm{cm}^{3}$. Besides gaseous tritium exposure the characteristics of the 3D diodes vis a vis the tritium absorbed in the near surface regions of the device, denoted surface tritium, is examined; additionally, these results are compared against the performance of the diode powered by tritium decay betas emanating from a scandium tritide foil.

The fabrication process of the 3D betavoltaic (BV) cells is described in Ref. 4. In those 3D cells, electrochemical anodized etching of $p$-type silicon leads to the formation of porous structures; subsequently, thermal diffusion process forms $p n$ junction over the entire planar and internal pore surfaces. The $50 \mathrm{~nm}$ thick aluminum contact layers are evaporated on backside and on top of the porous side. A scanning electron microscopy (SEM) picture of the crosssection of the porous diode and a schematic of the diode structure are shown as an inset of Fig. 1.

The test with gaseous tritium was performed using a prototype sample with a size of $1 \times 2 \mathrm{~cm}^{2}$ sealed in a stainless steel chamber. The tritium gas was gradually introduced into the chamber from vacuum level. The IV characteristics of the sample are monitored via appropriate electric feedthroughs during the pressurization. There are two different portions of tritium that contribute to the produced betapower. These are the tritium layer above the top planar surface and the tritium in the pores (see inset of Fig. 1). Due to self-absorption, beta particles at $5.7 \mathrm{keV}$ traverse approximately a centimeter through tritium gas at nominal pressure of $1 \mathrm{~atm}$ before substantially losing their kinetic energy. The 


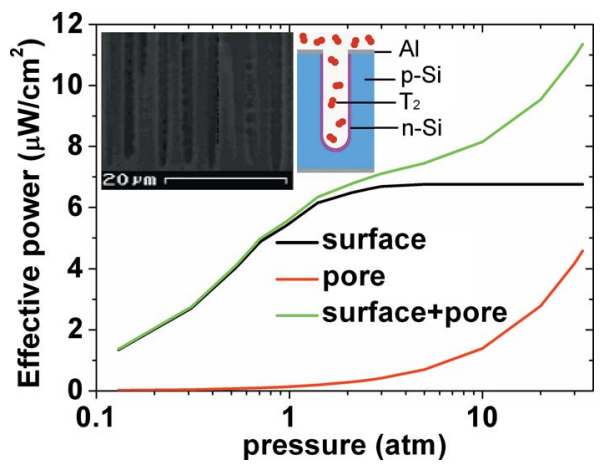

FIG. 1. (Color online) Calculated effective specific power from the tritium layer above the planar cell surface, the internal pores, and the total specific power, as a function of the tritium pressure. The inset shows a SEM image of the 3D silicon device (side view) and a schematic of the diode structure with illustration of tritium in the pore and on the planar cell surface.

self-attenuation length is approximately in inverse proportion to gas pressure. Hence, the beta flux reaching the top surface saturates at around $2 \mathrm{~atm}$ with a maximum power flux of $14 \mu \mathrm{W} / \mathrm{cm}^{2}{ }^{1}$. The betas reaching the un-anodized nonporous surface is estimated to lose $3 / 4$ of the beta energy due to the $50 \mathrm{~nm} \mathrm{Al}$ coating. ${ }^{7}$ Thus the maximum beta power flux reaching the top surface (anodized porous and unanodized nonporous regions) is estimated to be $6.8 \mu \mathrm{W} / \mathrm{cm}^{2}$. In sharp contrast, tritium gas trapped in the small porous structure suffers negligible self-absorption due to the small pore diameter of $\sim 1 \mu \mathrm{m}$, thus almost $100 \%$ of tritium decay beta particles produced within the pores impinge on the pore surface. The beta power flux therefore increases linearly with tritium pressure. It is estimated that the total void volume in the pores is $\sim 1.6 \mathrm{~mm}^{3}$ for a cell of $1 \mathrm{~cm}^{2}$; accordingly the power flux inside porous structure will increase linearly to $4.6 \mu \mathrm{W} / \mathrm{cm}^{2}$ at $33 \mathrm{~atm}$ tritium pressure. Figure 1 illustrates calculated effective specific beta power flux from the tritium layer above the cell and the internal pores as a function of tritium pressure. The effective power refers to the beta flux power actually impinging the cell/silicon-absorber, which is a fraction of the total power present, having excluded the geometry and attenuation losses. As shown in Fig. 1, only the tritium in the pores contributes to the total effective power for tritium pressures exceeding $2 \mathrm{~atm}$; this displays the advantage of the 3D diode structure.

The experimental IV characteristics, short circuit current density $\left(J_{\mathrm{sc}}\right)$ and open circuit voltage $\left(V_{\mathrm{oc}}\right)$, as a function of

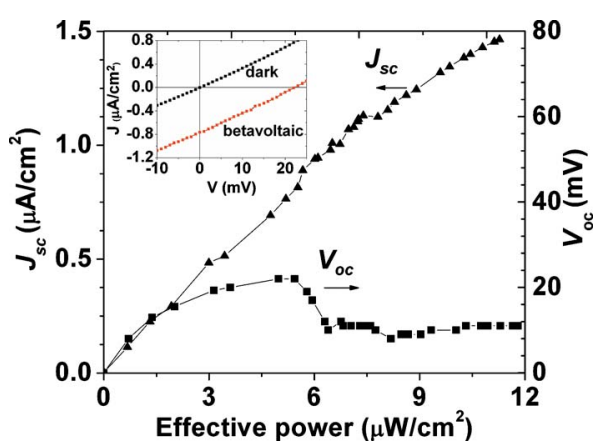

FIG. 2. (Color online) The short circuit current density $\left(J_{\mathrm{sc}}\right)$ and the open circuit voltage $\left(V_{\mathrm{oc}}\right)$ as a function of effective power impinging on the cell. The IV characteristics of the 3D diode under dark conditions without tritium (dark), and with tritium at a tritium pressure of 0.8 atm (betavoltaic) as an inset.

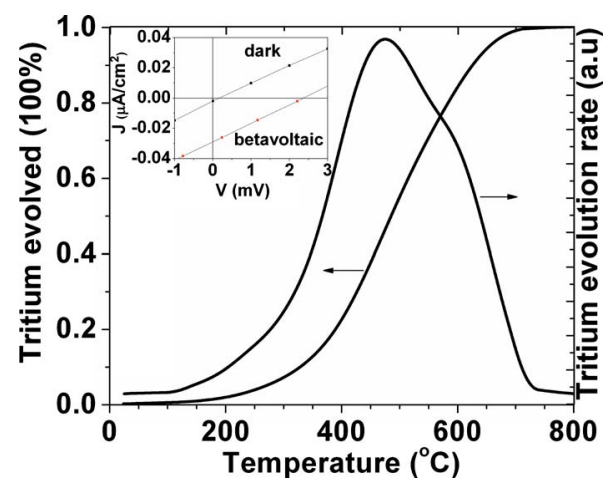

FIG. 3. (Color online) The normalized cumulative tritium effusion profile and the effusion rate as a function of temperature for the 3D silicon cell tritiated in tritium gas at $100{ }^{\circ} \mathrm{C}$ and 10 bar. The inset shows the IV characteristics of the 3D BV cell under dark conditions and when powered by tritium incorporated into the porous surface (direct solid tritium).

effective power impinging the cell of the 3D diodes are presented in Fig. 2. Typical IV curves of the diode in dark without tritium and with tritium at tritium pressure of $0.8 \mathrm{~atm}$ are shown as an inset. The $J_{\mathrm{sc}}$ increases nearly linearly with the effective power and reaches a maximum of $1.49 \mu \mathrm{A} / \mathrm{cm}^{2}$ at $33 \mathrm{~atm}$ tritium pressure. This observation validates the concept of $3 \mathrm{D}$ diode for nuclear power production. However, $V_{\text {oc }}$ does not behave as expected. The $V_{\text {oc }}$ reaches a maximum of $22 \mathrm{mV}$ at $0.8 \mathrm{~atm}$ and subsequently drops to $11 \mathrm{mV}$ when the pressure reaches $33 \mathrm{~atm}$. It is unclear what causes the drop in cell potential. The output power $\mathrm{P}_{\text {out }}$ also does not increase beyond 0.8 atm given the drop of $V_{\mathrm{oc}}$; the maximum power obtained is $9.2 \mathrm{nW}$ for the $1 \times 2 \mathrm{~cm}^{2}$ sample. The effective energy conversion efficiency $\left(\eta_{P}\right)$ increases from $0.03 \%(0.05 \mathrm{~atm})$ to $0.09 \%$ $(0.8 \mathrm{~atm})$, and does not increase further due to the voltage drop. It is noted that the efficiency here is calculated by using the effective power impinging the cell/silicon-absorber.

To further analyze the results obtained from the pressure experiment, the 3D diode was also powered by two different forms of solid tritium fuels. The porous surface of the 3D silicon cell is capable of absorbing tritium through high pressure tritium dissolution in the near surface regions, ${ }^{8}$ thereby offering a convenient 3D beta source to power the diode. We denote this as direct solid tritium fuelling of a betavoltaic device. The porous diode was tritiated in tritium gas at a slightly elevated temperature of $100{ }^{\circ} \mathrm{C}$ and a pressure of 10 bar for $10 \mathrm{~h}$. Tritium effusion measurement was carried out to determine the quantity and bonding characteristics of the

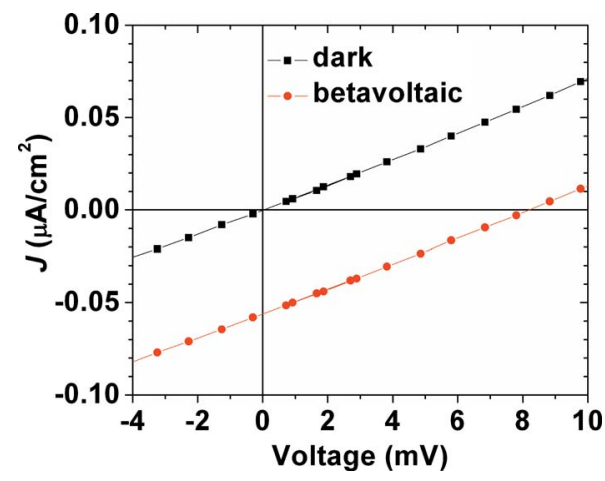

FIG. 4. (Color online) IV characteristics of the 3D betavoltaic cell under dark conditions and when powered by a ScT film (indirect solid tritium). 
TABLE I. Summary of the effective current collection and energy conversion efficiencies, and the specific current and power generation for the 3D porous betavoltaic device powered by different forms of tritium sources.

\begin{tabular}{|c|c|c|c|}
\hline & $\begin{array}{c}\text { Gaseous tritium at } \\
0.8 \mathrm{~atm}\end{array}$ & $\begin{array}{l}\text { Surface occluded } \\
\text { tritium source }\end{array}$ & ScT tritium source \\
\hline Effective current collection efficiency $(\%)^{\mathrm{a}}$ & 56 & 82 & 86 \\
\hline Effective energy conversion efficiency $(\%)^{\mathrm{a}}$ & 0.09 & 0.013 & 0.05 \\
\hline Specific current generation $(\mu \mathrm{A} / \mathrm{Ci})^{\mathrm{b}}$ & 0.71 & 7.25 & 0.92 \\
\hline Specific power generation $(\mathrm{nW} / \mathrm{Ci})^{\mathrm{b}}$ & 2.21 & 4.35 & 2 \\
\hline
\end{tabular}

${ }^{a}$ Effective current collection and power generation efficiencies are calculated based on the effective beta flux energy impinging the cell/silicon-absorber.

${ }^{\mathrm{b}}$ Specific current generation and power generation are calculated based on the total tritium activity present.

tritium occluded in the near surface regions of the device., The tritium effusion rate profile (Fig. 3) shows the outdiffusion peak around $480{ }^{\circ} \mathrm{C}$. The effusion profiles are consistent with previously reported results of tritiation of the crystalline silicon surface. ${ }^{8}$ For the $1 \times 1 \mathrm{~cm}^{2}$ porous 3D structure, the effusion experiment yielded $4 \mathrm{mCi}$ of total tritium activity. Considering the 50-fold magnification in the surface area of the porous sample, the corresponding surface activity is $0.08 \mathrm{mCi} / \mathrm{cm}^{2}$. This result is consistent with a reported value of $0.1 \mathrm{mCi} / \mathrm{cm}^{2}$ for crystalline silicon tritiated under similar conditions. ${ }^{8}$ These measurements confirm that tritium has been occluded in the sidewall surfaces of the porous 3D diode. The IV characteristics of the diode before and after the tritiation are shown as an inset. A $J_{\mathrm{sc}}$ of $29 \mathrm{nA} / \mathrm{cm}^{2}$ and a $V_{\mathrm{oc}}$ of $2.4 \mathrm{mV}$ was measured for the tritiated cell. The measured $V_{\text {oc }}$ is relatively low due to the lower power input; $4 \mathrm{mCi} / \mathrm{cm}^{2}$ is equivalent to a power density of $0.13 \mu \mathrm{W} / \mathrm{cm}^{2}$. The effective current collection efficiency and effective energy conversion efficiency are calculated to be $82 \%$ and $0.013 \%$, respectively. The foregoing experiment is essentially equivalent to that of a cell powered by tritium gas present only in the pores.

As a comparison to the two-dimensional power configuration, a $300 \mathrm{~nm}$ thick scandium tritide (ScT) film with a surface activity of $15 \mathrm{mCi} / \mathrm{cm}^{2},{ }^{10}$ which is equivalent to a power density of $0.5 \mu \mathrm{W} / \mathrm{cm}^{2}$, was used to power the $3 \mathrm{D}$ diode; we denote this as in-direct solid tritium fuelling of a betavoltaic device. This experiment is essentially equivalent to that of a cell powered by tritium gas present only above the planar surface (and not in the pores). The measurement was performed at standard conditions with the ScT film directly in contact with the diode. A $J_{\mathrm{sc}}$ of $55 \mathrm{nA} / \mathrm{cm}^{2}$ and a $V_{\text {oc }}$ of $8 \mathrm{mV}$ was measured, resulting in a maximum output power of $0.12 \mathrm{nW} / \mathrm{cm}^{2}$. The IV characteristics of the cell measured in the dark with ScT beta irradiation are shown in Fig. 4. Taking into account the shielding effect of $50 \mathrm{~nm} \mathrm{Al}$ contact layer, the input isotope power flux reaching the device is estimated to be $240 \mathrm{nW} / \mathrm{cm}^{2}$. The maximum short circuit density is calculated to be $64 \mathrm{nA} / \mathrm{cm}^{2}$, which leads to an effective current collection efficiency of $86 \%$. The effective energy conversion efficiency is calculated to be $0.05 \%$. The above values are based on the effective activity impinging the cell. Due to the self-absorption and geometry losses $\left(\sim 3 / 4\right.$ in total), the overall efficiency will be discounted. ${ }^{7}$
Table I summarizes the effective current collection and energy conversion efficiencies for different forms of sources, as well as the specific current and power generation which is calculated per unit curie of tritium in each case. It indicates that the surface occluded tritium source provides the highest current and power generation per curie since self-absorption and geometry loss are avoided in this configuration.

In summary, the power scaling performance of the 3D betavoltaic diode was studied in this letter. The IV characteristics of the 3D diode under high tritium pressure show a continuous increase in short-circuit current as a function of tritium pressure in contrast to current saturation for a planar diode. The study suggests that power production can be improved using 3D pn-junction with high pressure tritium or equivalently with direct occlusion of high density tritium in the near surface regions of the device. The stability of tritium occlusion in semiconductor materials will have to be investigated as part of the development of practical power conversion devices.

This work was supported by NSF Grant No. 0826289 and NSERC grants. The authors gratefully acknowledge Dr. Larry L. Gadeken of Betabatt Inc. for providing the prototype $3 \mathrm{D}$ porous silicon cells.

${ }^{1}$ K. E. Bower, Y. A. Barbanel, Y. G. Shreter, and G. W. Bohnert, Polymer, Phosphors, and Voltaics for Radioisotope Microbatteries (CRC, Boca Raton, 2002).

${ }^{2}$ T. Kosteski, N. P. Kherani, P. Stradins, F. Gaspari, W. T. Shmayda, L. S. Sidhu, and S. Zukotynski, IEE Proc.: Circuits Devices Syst. 150, 274 (2003).

${ }^{3}$ M. V. S. Chandrashekhar, C. I. Thomas, H. Li, M. G. Spencer, and A. Lal, Appl. Phys. Lett. 88, 033506 (2006).

${ }^{4}$ W. Sun, N. P. Kherani, K. D. Hirschman, L. L. Gadeken, and P. M. Fauchet, Adv. Mater. (Weinheim, Ger.) 17, 1230 (2005).

${ }^{5}$ R. Duggirala, S. Tin, and A. Lal, TRANSDUCERS 2007 International Solid-State Sensors, Actuators and Microsystems Conference, 2007 (unpublished), pp. 279-282.

${ }^{6}$ B. Liu, K. P. Chen, N. P. Kherani, S. Zukotynski, and A. B. Antoniazzi, Appl. Phys. Lett. 88, 134101 (2006).

${ }^{7}$ N. P. Kherani and W. T. Shmayda, Fusion Technol. 21, 334 (1992).

${ }^{8}$ B. Liu, K. P. Chen, N. P. Kherani, T. Kosteski, S. Costea, S. Zukotynski, and A. B. Antoniazzi, Appl. Phys. Lett. 89, 044104 (2006).

${ }^{9}$ N. P. Kherani, B. Liu, K. Virk, T. Kosteski, F. Gaspari, W. T. Shmayda, S. Zukotynski, and K. P. Chen, J. Appl. Phys. 103, 024906 (2008).

${ }^{10}$ B. Liu, K. P. Chen, N. P. Khernai, S. Zukotynski, and A. B. Antoniazzi, Appl. Phys. Lett. 92, 083511 (2008). 
Applied Physics Letters is copyrighted by the American Institute of Physics (AIP). Redistribution of journal material is subject to the AIP online journal license and/or AIP copyright. For more information, see http://ojps.aip.org/aplo/aplcr.jsp 